\title{
Myanmar Early Warning Score (MEWS): The introduction of a standardised approach to vital sign monitoring at New Yangon General Hospital in Myanmar
}

\author{
Authors: Theeba Krishnamoorthy, ${ }^{\mathrm{A}, \mathrm{B}}$ Aye Myitzu, ${ }^{\mathrm{C}, \mathrm{D}}$ Zaw Lynn Aung ${ }^{\mathrm{C}, \mathrm{D}}$ and Russell Roberts ${ }^{\mathrm{E}, \mathrm{F}}$
}

\begin{abstract}
Aims
To pilot the introduction of a standardised 'track and trigger' early warning score for the recording of bedside observations at New Yangon General Hospital (NYGH) in Yangon, Myanmar.
\end{abstract}

\section{Methods}

As part of the scoping exercise for a quality improvement (QI) project aimed at improving the early management of acute kidney injury (AKI) we noticed that there were deficiencies in the recording of bedside observations in our partner institution NYGH.

Our initial audit revealed that five different observation charts were in use with multiple charts used for $80 \%$ of patients. We noted many missing observations ( $23 \%$ in our survey) and inconsistency in responsibility for vital sign recording. Finally, we discovered variation in awareness of 'normal' ranges for vital signs. Results from our initial audit were shared with the NYGH team as our rationale for change.

In the UK, the Royal College of Physicians has led development of the National Early Warning Score (NEWS) which standardises vital sign charting and uses a scoring system to facilitate a 'track and trigger' approach. A physiological 'track and trigger' warning system is novel to Myanmar. We decided to pilot the introduction of NEWS at NYGH.

Our strategy involved data collection, NEWS teaching sessions in Burmese and dissemination of bilingual educational material via 'Beware AKI' Facebook page and posters. This was then followed with the launch of our first version of the NEWS chart in the six bed female high dependency unit, with our assistant surgeons completing the charts. Regular audits were carried out, with feedback being delivered to the team via Facebook Messenger. The day-to-day running of the chart was supported by a local NEWS lead and champion. We had repeated cycles of design modification supported by focus groups and questionnaires that

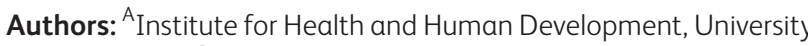

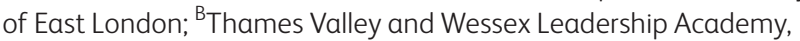
London, UK; ' ${ }^{C}$ Department of Medicine, University of Medicine 1, Myanmar; ${ }^{D}$ New Yangon General Hospital, Yangon, Myanmar;

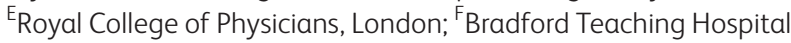
NHS Trust, Bradford, UK
}

led to the creation of the new Myanmar Early Warning Score (MEWS) chart. The major design modification was widening of the temperature box to better illustrate temperature swings in tropical infectious diseases. We also designed electronic and hard copy teaching materials on MEWS implementation to support spread across Myanmar. MEWS is currently rolled out in the medical unit, with spread to surgery imminent.

\section{Results}

In the short term, we demonstrated successful recording of all six vital signs on a NEWS chart for increasing numbers of beds with approximately 100 patients exposed to date. We also demonstrated through regular audit increased awareness among staff of the significance of abnormal vital signs. We have delivered teaching to a total of 100 doctors and nurses and noted improvements in levels of knowledge around abnormal vital signs.

\section{Conclusion}

We have successfully introduced the use of a 'track and trigger' vital signs chart at NYGH in Myanmar. Having modified the chart in response to local feedback we have shown that introduction of the MEWS chart has eliminated much of the variation in vital sign recording and reduced the number of missed observations. From running this project we learnt the importance of starting the project with small-scale initial intervention supported by multidisciplinary education as we were challenging established practice in a hierarchical environment. Secondly, we discovered that in Myanmar social media was the best way of communicating with project participants and seeking feedback. In our opinion, MEWS modified for Myanmar (and tropical climates) facilitates a standardised approach to vital signs and acts as a physiological 'track and trigger' warning system. Adoption of MEWS should improve efficiency in bedside observations with potential benefit for patient outcomes and should facilitate further QI work in Myanmar's hospitals. Finally, we have demonstrated that a smallscale QI approach supported by education and the use of social media can change established practice at the individual ward leve without additional resources.

\section{Conflict of interest statement}

Funded by the Brighter Future Foundation. 